\title{
A Nonoverlapping Domain Decomposition Method for an Exterior Anisotropic Quasilinear Elliptic Problem in Elongated Domains
}

\author{
Baoqing Liu and Qikui Du \\ Jiangsu Key Laboratory for NSLSCS, School of Mathematical Sciences, Nanjing Normal University, Nanjing, \\ Jiangsu 210023, China \\ Correspondence should be addressed to Qikui Du; duqikui@njnu.edu.cn
}

Received 2 September 2012; Accepted 17 December 2012

Academic Editor: Yong-Kui Chang

Copyright (C) 2013 B. Liu and Q. Du. This is an open access article distributed under the Creative Commons Attribution License, which permits unrestricted use, distribution, and reproduction in any medium, provided the original work is properly cited.

Based on the Kirchhoff transformation, a nonoverlapping domain decomposition method is discussed for solving exterior anisotropic quasilinear problems with elliptic artificial boundary. By the principle of the natural boundary reduction, we obtain the natural integral equation for the anisotropic quasilinear problem on elliptic artificial boundaries and construct the algorithm and analyze its convergence. Moreover, we give the existence and uniqueness result for the original problem. Finally, some numerical examples are presented to illustrate the feasibility of the method.

\section{Introduction}

When solving a problem modelled by a linear or nonlinear partial differential equation in the bounded or unbounded domain, domain decomposition methods are one of the most efficient techniques. one can refer to [1-4] and references therein for more details. Based on natural boundary reduction $[5,6]$, the overlapping and nonoverlapping domain decomposition methods can be viewed as effective ways to solve the problems in the unbounded domains. These techniques have been used to solve many linear problems $[6-8]$, and they have also been generalized to linear or nonlinear wave problems $[5,9,10]$. In this paper, we consider a nonoverlapping domain decomposition method for an exterior anisotropic quasilinear elliptic problem with elliptical artificial boundary. By the Kirchhoff transformation, we will discuss some exterior anisotropic quasilinear elliptic problems [11-15] by virtue of the nonoverlapping domain decomposition method.

Let $\Omega$ be an elongated, bounded, and simple connected domain in $\mathbb{R}^{2}$ with sufficiently smooth boundary $\partial \Omega=\Gamma_{0}$,
$\Omega^{c}=\mathbb{R}^{2} / \bar{\Omega}$. We consider the numerical solution to the exterior anisotropic quasilinear problem

$$
\begin{aligned}
& -\left(\frac{\partial}{\partial x}\left(\alpha a(\mathbf{x}, u) \frac{\partial u}{\partial x}\right)+\frac{\partial}{\partial y}\left(\beta a(\mathbf{x}, u) \frac{\partial u}{\partial y}\right)\right)=f(\mathbf{x}), \\
& \text { in } \Omega^{c} \text {, } \\
& u=0, \quad \text { on } \Gamma_{0}, \\
& u(\mathbf{x})=\mathscr{O}(1), \quad \text { as }|\mathbf{x}| \longrightarrow \infty,
\end{aligned}
$$

with $\beta>\alpha>0$ or $\alpha=\beta=1, \mathbf{x}=(x, y), a(\cdot, \cdot)$, and $f(\mathbf{x})$ are the given functions which will be ranked as below.

Problem (1) has many physical applications in, for example, the field of heat transfer, where $a$ is the thermal conductivity of the medium and $u$ is the temperature field; the field of compressible flow, where $a$ is the density and $u$ is the velocity potential. Problem (1) can also describe a temperature distribution in large transformers whose magnetic cores (consisting of iron tins) are nonlinear anisotropic media 


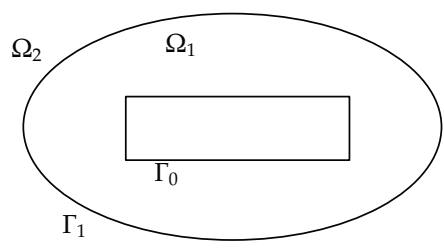

FIgURE 1: The illustration of domains $\Omega_{1}$ and $\Omega_{2}$.

where $a$ is the heat conductivity. One can also refer to [11$14,16]$ for more details.

Following $[11,13]$, suppose that the given function $a(\cdot, \cdot)$ satisfies

$$
0<C_{0} \leq a(\mathbf{x}, u) \leq C_{1}
$$

$\forall u \in \mathbb{R}$, and for almost all $\mathbf{x} \in \Omega^{c}$,

Where the two constants $C_{0}, C_{1} \in \mathbb{R}$. In the following, we suppose that the function $f(\mathbf{x}) \in L^{2}\left(\Omega^{c}\right)$ has compact support, that is, there exists a constant $\gamma_{0}>0$, such that

$$
\text { supp } f \subset \Omega_{\gamma_{0}}=\left\{\mathbf{x} \in \mathbb{R}^{2}|| \mathbf{x} \mid \leq \gamma_{0}\right\} \text {. }
$$

We also assume that

$$
a(\mathbf{x}, u) \doteq a_{0}(u), \quad \text { when }|\mathbf{x}| \geq \gamma_{0} .
$$

Now, we introduce an elliptical artificial boundary $\Gamma_{1}$ enclosing $\Gamma_{0}$ such that

$$
\begin{gathered}
\Gamma_{1}=\left\{(\mu, \varphi) \mid \mu=\mu_{1}>\gamma_{0}, 0 \leq \varphi \leq 2 \pi\right\} \\
\text { and } \operatorname{dist}\left(\Gamma_{1}, \Gamma_{0}\right)=\delta_{0}>0
\end{gathered}
$$

Then, $\Omega^{c}$ is divided into two nonoverlapping subdomains $\Omega_{1}$ and $\Omega_{2}$ (see Figure 1), where $\Omega_{1}$ denotes the bounded domain between $\Gamma_{0}$ and $\Gamma_{1}$, and $\Omega_{2}$ refers to the unbounded domain outside $\Gamma_{1}$. The original problem (1) is decomposed into two subproblems in domains $\Omega_{1}$ and $\Omega_{2}$ with $\Omega_{1} \cap$ $\Omega_{2}=\emptyset$. We have the nonoverlapping domain decomposition algorithm.

Step 1. Choose an initial value $\lambda^{0} \in H^{1 / 2}\left(\Gamma_{1}\right)$, and put $k=0$.

Step 2. Solve a Dirichlet boundary value problem in the exterior domain $\Omega_{2}$ :

$$
\begin{gathered}
-\left(\frac{\partial}{\partial x}\left(\alpha a\left(\mathbf{x}, u_{2}^{k}\right) \frac{\partial u_{2}^{k}}{\partial x}\right)+\frac{\partial}{\partial y}\left(\beta a\left(\mathbf{x}, u_{2}^{k}\right) \frac{\partial u_{2}^{k}}{\partial y}\right)\right) \\
=0, \quad \text { in } \Omega_{2}, \\
u_{2}^{k}=\lambda^{k}, \quad \text { on } \Gamma_{1}, \\
u_{2}^{k}(\mathbf{x})=\mathcal{O}(1), \quad \text { as }|\mathbf{x}| \longrightarrow \infty .
\end{gathered}
$$

Step 3. Solve a mixed boundary value problem in the interior domain $\Omega_{1}$ :

$$
\begin{gathered}
-\left(\frac{\partial}{\partial x}\left(\alpha a\left(\mathbf{x}, u_{1}^{k}\right) \frac{\partial u_{1}^{k}}{\partial x}\right)+\frac{\partial}{\partial y}\left(\beta a\left(\mathbf{x}, u_{1}^{k}\right) \frac{\partial u_{1}^{k}}{\partial y}\right)\right)=f(\mathbf{x}) \\
\text { in } \Omega_{1} \\
\frac{\partial u_{1}^{k}}{\partial n_{1}}=-\frac{\partial u_{2}^{k}}{\partial n_{2}}, \quad \text { on } \Gamma_{1}, \\
u_{1}^{k}=0, \quad \text { on } \Gamma_{0} .
\end{gathered}
$$

Step 4. Update the boundary value $0<\theta_{k}<1$,

$$
\lambda^{k+1}=\theta_{k} u_{1}^{k}+\left(1-\theta_{k}\right) \lambda^{k}, \quad \text { on } \Gamma_{1} \text {. }
$$

Step 5. Put $k=k+1$, turn to Step 2 .

The relaxation factor $\theta_{k}$ is a suitably chosen real number. Notice that, in Step 3, we solve problem (8) by the standard finite element method and only need the normal derivative of the solution to problem (7) in Step 2. So, we need not to solve (7) directly. Based on the Kirchhoff transformation, the natural integral equation for the quasilinear problem can be obtained by the natural boundary reduction $[6,17,18]$. Particularly, when $a(\mathbf{x}, u)=c$ which is independent of $\mathbf{x}$ and $u,[6,8,19]$ have discussed the corresponding problems by this technique. Now, we introduce the so-called Kirchhoff transformation [20]

$$
w(\mathbf{x})=\int_{0}^{u(\mathbf{x})} a(\xi) d \xi, \quad \mathbf{x} \in \Omega^{c} .
$$

Then, we have

$$
\begin{gathered}
\nabla w=a(u) \nabla u \\
\left(\alpha \frac{\partial w}{\partial x}, \beta \frac{\partial w}{\partial y}\right)=\left(\alpha a(u) \frac{\partial u}{\partial x}, \beta a(u) \frac{\partial u}{\partial y}\right) .
\end{gathered}
$$

From (7), we have that $w$ satisfies the following problem

$$
\begin{gathered}
-\left(\alpha \frac{\partial^{2} w^{k}}{\partial x^{2}}+\beta \frac{\partial^{2} w^{k}}{\partial y^{2}}\right)=0, \quad \text { in } \Omega_{2}, \\
w^{k}=\int_{0}^{\lambda^{k}} a_{0}(\xi) d \xi, \quad \text { on } \Gamma_{1} .
\end{gathered}
$$

The rest of the paper is organized as follows. In Section 2, we obtain the natural integral equation for the quasilinear problem in the elliptical unbounded domain. In Section 3, we state the nonoverlapping domain decomposition method and discuss the convergence of the algorithm. We also show the existence and uniqueness of the original problem. At last, in Section 4, we present some numerical examples to illustrate the efficiency and feasibility of our method. 


\section{Natural Boundary Reduction}

In this section, by virtue of the Poisson integral formula and natural integral equation for the linear problem, we will obtain the corresponding results for the quasilinear problem in $\Omega_{2}$. For this purpose, we need to discuss some properties between elliptic coordinates $(\mu, \varphi)$ and Cartesian coordinates $(x, y)$ first. The relationship between the two coordinates can be expressed as below

$$
\begin{aligned}
& x=f_{0} \cosh \mu \cos \varphi, \\
& y=f_{0} \sinh \mu \sin \varphi,
\end{aligned}
$$

with $f_{0}=\sqrt{a^{2}-b^{2}}, a=f_{0} \cosh \mu_{1}$, and $b=f_{0} \sinh \mu_{1}$. Following from [18], we have the following.

Theorem 1. The transformation between elliptic coordinates and Cartesian coordinates in (14) possesses the following property.

(1) The Jacobian determinant of (14) is

$$
\begin{gathered}
J=f_{0}^{2} \cosh ^{2} \mu \sin ^{2} \varphi+f_{0}^{2} \sinh ^{2} \mu \cos ^{2} \varphi \\
=f_{0}^{2}\left(\cosh ^{2} \mu-\cos ^{2} \varphi\right), \\
J=0 \text { if and only if }(x, y)=\left( \pm f_{0}, 0\right) .
\end{gathered}
$$

(2) For $u \in C^{2}\left(\mathbb{R}^{2}\right)$, the following holds

$$
\frac{\partial^{2} u}{\partial \mu^{2}}+\frac{\partial^{2} u}{\partial \varphi^{2}}=J\left(\frac{\partial^{2} u}{\partial x^{2}}+\frac{\partial^{2} u}{\partial y^{2}}\right)
$$

(3) For the exterior domain $\Omega_{2}$, we have

$$
\frac{\partial u}{\partial v}=-\frac{1}{\sqrt{J}} \frac{\partial u}{\partial \mu}
$$

where $v$ refers to the unit exterior normal vector on $\Gamma_{\mu_{1}}$ (regarded as the inner boundary of $\Omega_{2}$ ).

Proof. The conclusions 1 and 2 can be obtained by direct computation. And 3 follows from the property

$$
v=-\frac{1}{\sqrt{J}}\left(f_{0} \sinh \mu \cos \varphi, f_{0} \cosh \mu \sin \varphi\right) .
$$

2.1. Natural Integral Equation for $\alpha=\beta=1$. Assume that $w(\mathbf{x})$ is the solution to problem (13) and the value $\left.w\right|_{|\mu|=\mu_{1}}$ is given, that is,

$$
\left.w\right|_{|\mu|=\mu_{1}}=w_{0}(\varphi) .
$$

Then, based on the natural boundary reduction, there are the Poisson integral formulas

$$
\begin{array}{r}
w(\mu, \varphi)=\frac{\mathrm{e}^{2 \mu}-\mathrm{e}^{2 \mu_{1}}}{2 \pi} \\
\times \int_{0}^{2 \pi} \frac{w_{0}\left(\varphi^{\prime}\right)}{\mathrm{e}^{2 \mu}+\mathrm{e}^{2 \mu_{1}}-2 \mathrm{e}^{\mu+\mu_{1}} \cos \left(\varphi-\varphi^{\prime}\right)} d \varphi^{\prime}, \\
\mu>\mu_{1},
\end{array}
$$

or

$$
\begin{aligned}
w(\mu, \varphi)= & \frac{1}{\pi} \sum_{j=1}^{\infty} \mathrm{e}^{j\left(\mu_{1}-\mu\right)} \\
& \times \int_{0}^{2 \pi} \cos j\left(\varphi-\varphi^{\prime}\right) w_{0}\left(\varphi^{\prime}\right) \\
& +\frac{1}{2 \pi} \int_{0}^{2 \pi} w_{0}\left(\varphi^{\prime}\right) d \varphi^{\prime}, \quad \mu>\mu_{1},
\end{aligned}
$$

and the natural integral equation

$$
\frac{\partial w}{\partial n}=\frac{1}{\sqrt{J_{0}}}\left[-\frac{1}{4 \pi \sin ^{2}(\varphi / 2)} * w_{0}(\varphi)\right], \quad \mu=\mu_{1},
$$

or

$$
\begin{aligned}
\frac{\partial w}{\partial n}= & \frac{1}{\pi \sqrt{J_{0}}} \sum_{j=1}^{\infty} j \\
& \times \int_{0}^{2 \pi} \cos j\left(\varphi-\varphi^{\prime}\right) w_{0}\left(\mu_{1}, \varphi^{\prime}\right) d \varphi, \\
\mu & =\mu_{1},
\end{aligned}
$$

the definition of $J_{0}$ can be found in the following. The Poisson integral formulas (20)-(21) and the natural integral equation (22)-(23) can also be expressed in the Fourier series forms

$$
\begin{gathered}
w(\mu, \varphi)=\sum_{j=-\infty}^{+\infty} a_{j} \mathrm{e}^{|j|\left(\mu_{1}-\mu\right)+\mathrm{i} j \varphi}, \quad \mu>\mu_{1}, \\
\frac{\partial w}{\partial n}=\frac{1}{\sqrt{J_{0}}} \sum_{j=-\infty}^{+\infty}|j| a_{j} \mathrm{e}^{\mathrm{i} j \varphi}, \quad \mu=\mu_{1},
\end{gathered}
$$

with $a_{j}=(1 / 2 \pi) \int_{0}^{2 \pi} w_{0}\left(\varphi^{\prime}\right) \mathrm{e}^{-\mathrm{i} j \varphi^{\prime}} d \varphi^{\prime}, J_{0}=f_{0}^{2}\left(\cosh ^{2} \mu_{1} \sin ^{2} \varphi+\right.$ $\left.\sinh ^{2} \mu_{1} \cos ^{2} \varphi\right)=f_{0}^{2}\left(\cosh ^{2} \mu_{1}-\cos ^{2} \varphi\right), \mathrm{i}=\sqrt{-1}$.

From (12), we obtain

$$
\frac{\partial w}{\partial n}=a_{0}(u) \frac{\partial u}{\partial n}
$$

Combining (11), (25), and (26), we get the exact artificial boundary condition of $u$ on $\Gamma_{\mu_{1}}$

$$
\begin{aligned}
& \left.a_{0}(u) \frac{\partial u(\mu, \varphi)}{\partial n}\right|_{\mu=\mu_{1}} \\
& =\frac{1}{\sqrt{J_{0}}} \sum_{j=-\infty}^{+\infty}|j| a_{j} \mathrm{e}^{\mathrm{i} j \varphi} \\
& =\frac{1}{\pi \sqrt{J_{0}}} \sum_{j=1}^{+\infty} j \int_{0}^{2 \pi} \cos j\left(\varphi-\varphi^{\prime}\right)\left(\int_{0}^{u\left(\mu_{1}, \varphi\right)} a_{0}(y) d y\right) d \varphi \\
& \doteq \mathscr{K}_{1}\left(u\left(\mu_{1}, \varphi\right)\right),
\end{aligned}
$$

with $a_{j}=(1 / 2 \pi) \int_{0}^{2 \pi}\left(\int_{0}^{u\left(\mu_{1}, \varphi^{\prime}\right)} a_{0}(y) d y\right) \mathrm{e}^{-\mathrm{i} j \varphi^{\prime}} d \varphi^{\prime}, J_{0}=f_{0}^{2}$ $\left(\cosh ^{2} \mu_{1}-\cos ^{2} \varphi\right), \mathrm{i}=\sqrt{-1}$. 
2.2. Natural Integral Equation for $\beta>\alpha>0$. Now, we assume that $\Gamma_{\mu_{1}}$ can be expressed in the form: $\Gamma_{\mu_{1}}=\left\{(x, y) \mid p x^{2}+\right.$ $\left.q y^{2}=R^{2}\right\}$, with $\beta q>\alpha p>0$. We also assume that $w(x)$ is the solution to problem (13) and the value $\left.w\right|_{|\mu|=\mu_{1}}$ is given, namely,

$$
\left.w\right|_{|\mu|=\mu_{1}}=w_{0}(\varphi) .
$$

Let $x=\sqrt{\alpha} \xi$ and $y=\sqrt{\beta} \eta$. Then, the boundary $\Gamma_{\mu_{1}}$ is changed by the elliptic boundary $\widetilde{\Gamma}_{1}=\left\{(\xi, \eta) \mid \alpha p \xi^{2}+\beta q \eta^{2}=R^{2}\right\}$; the unit exterior normal vector on $\widetilde{\Gamma}_{1}$ is

$$
v=-\frac{(\sqrt{\alpha p} \cos \theta, \sqrt{\beta q} \sin \theta)}{\sqrt{\alpha p \cos ^{2} \theta+\beta q \sin ^{2} \theta}} .
$$

By the above transformation, problem (13) changes into

$$
\begin{gathered}
-\left(\frac{\partial^{2} w}{\partial \xi^{2}}+\frac{\partial^{2} w}{\partial \eta^{2}}\right)=0, \quad \text { in } \widetilde{\Omega}_{2}, \\
w=w_{0}, \quad \text { on } \widetilde{\Gamma}_{1} .
\end{gathered}
$$

This is the right problem we talked in Section 2.1. Similar with (14), we let

$$
\xi=f_{0} \cosh \mu \cos \varphi, \quad \eta=f_{0} \sinh \mu \sin \varphi,
$$

with

$$
\begin{gathered}
f_{0}=\sqrt{\frac{\beta q-\alpha p}{\alpha p \beta q}} R, \\
\mu_{0}=\ln \left(\frac{\sqrt{\beta q}+\sqrt{\alpha p}}{\sqrt{\beta q-\alpha p}}\right), \\
\widetilde{\Gamma}_{1}=\left\{(\mu, \varphi) \mid \mu=\mu_{0}, \varphi \in[0,2 \pi]\right\}, \\
\widetilde{\Omega}_{2}=\left\{(\mu, \varphi) \mid \mu>\mu_{0}, \varphi \in[0,2 \pi]\right\} .
\end{gathered}
$$

Then, just the same as the problem discussed in Section 2.1, we have the natural integral equation on $\Gamma_{\mu_{1}}$

$$
\begin{aligned}
& \alpha n_{x} \frac{\partial w}{\partial x}+\beta n_{y} \frac{\partial w}{\partial y} \\
& \quad=-\sqrt{\frac{\alpha p \beta q}{p \cos ^{2} \varphi+q \sin ^{2} \varphi}}\left[\frac{1}{4 \pi R \sin ^{2}(\varphi / 2)} * w_{0}(\varphi)\right],
\end{aligned}
$$

where $\left(n_{x}, n_{y}\right)=(x / R, y / R)$ is the unit exterior normal vector on $\Gamma_{\mu_{1}}$. From (13), we obtain

$$
\alpha n_{x} \frac{\partial w}{\partial x}+\beta n_{y} \frac{\partial w}{\partial y}=\alpha n_{x} a_{0}(u) \frac{\partial u}{\partial x}+\beta n_{y} a_{0}(u) \frac{\partial u}{\partial y} .
$$

Combining (11), (34), and (35), we obtain the exact artificial boundary condition of $u$ on $\Gamma_{\mu_{1}}$

$$
\begin{aligned}
&\left.\left(\alpha n_{x} a_{0}(u) \frac{\partial u}{\partial x}+\beta n_{y} a_{0}(u) \frac{\partial u}{\partial y}\right)\right|_{\mu=\mu_{1}} \\
&=-\sqrt{\frac{\alpha p \beta q}{p \cos ^{2} \varphi+q \sin ^{2} \varphi}} \\
& \times\left[\frac{1}{4 \pi R \sin ^{2}(\varphi / 2)} *\left(\int_{0}^{u\left(\mu_{1}, \varphi\right)} a_{0}(y) d y\right)\right] \\
&= \sqrt{\frac{\alpha p \beta q}{p \cos ^{2} \varphi+q \sin ^{2} \varphi}} \sum_{j=-\infty}^{+\infty} \frac{j}{2 \pi R} \\
& \quad \times \int_{0}^{2 \pi} \cos j\left(\varphi-\varphi^{\prime}\right)\left(\int_{0}^{u\left(\mu_{1}, \varphi\right)} a_{0}(y) d y\right) d \varphi \\
& \doteq \mathscr{K}_{1}\left(u\left(\mu_{1}, \varphi\right)\right) .
\end{aligned}
$$

\section{Variational Problem and Convergence Analysis of the Algorithm}

3.1. The Equivalent Variational Problem. Now, we consider problem (8). We will use $W^{m, p}$ denoting the standard Sobolev spaces. $\|\cdot\|$ and $|\cdot|$ refer to the corresponding norms and seminorms. Especially, we define $H^{m}(\Omega)=W^{m, 2}(\Omega)$, $\|$. $\left\|_{m, \Omega}=\right\| \cdot \|_{m, 2, \Omega}$, and $|\cdot|_{m, \Omega}=|\cdot|_{m, 2, \Omega}$. Let us introduce the space

$$
V=\left\{v \in H^{1}\left(\Omega_{1}\right)|v|_{\Gamma_{0}}=0\right\},
$$

and the corresponding norms

$$
\begin{gathered}
\|v\|_{0, \Omega_{1}}=\sqrt{\int_{\Omega_{1}}|v|^{2} d \mathbf{x},} \\
\|v\|_{1, \Omega_{1}}=\sqrt{\int_{\Omega_{1}}\left(|v|^{2}+|\nabla v|^{2}\right) d \mathbf{x} .}
\end{gathered}
$$

The boundary value problem (8) is equivalent to the following variational problem.

Find $u \in V$, such that

$$
D(u ; u, v)+\widehat{D}(u ; u, v)=F(v), \quad \forall v \in V,
$$

with

$$
\begin{aligned}
D(t ; u, v)= & \int_{\Omega_{1}} a(\mathbf{x}, t)\left(\alpha \frac{\partial u}{\partial x} \frac{\partial v}{\partial x}+\beta \frac{\partial u}{\partial y} \frac{\partial v}{\partial y}\right) d \mathbf{x} \\
\widehat{D}(t ; u, v)= & \sum_{j=1}^{+\infty} \frac{\sqrt{\alpha \beta}}{j \pi} \times \int_{0}^{2 \pi} \int_{0}^{2 \pi} a_{0}\left(t\left(\mu_{1}, \varphi^{\prime}\right)\right) \\
& \times \frac{\partial u\left(\mu_{1}, \varphi^{\prime}\right)}{\partial \varphi^{\prime}} \frac{\partial v\left(\mu_{1}, \varphi\right)}{\partial \varphi} \\
& \times \cos j\left(\varphi^{\prime}-\varphi\right) d \varphi^{\prime} d \varphi
\end{aligned}
$$


where $\widehat{D}(t ; u, v)$ follows from Green's formula, (27) with $d s=$ $\sqrt{J_{0}} d \varphi$, and (36) with $d s=(R / \sqrt{p q}) \sqrt{p \cos ^{2} \varphi+q \sin ^{2} \varphi} d \varphi$.

And,

$$
F(v)=\int_{\Omega_{1}} f(\mathbf{x}) v(\mathbf{x}) d \mathbf{x}
$$

3.2. Convergence Analysis of the Method in Continuous Case. From (10)-(11), the original problem (1) can be changed to

$$
\begin{gathered}
-\left(\alpha \frac{\partial^{2} w}{\partial x^{2}}+\beta \frac{\partial^{2} w}{\partial y^{2}}\right)=f(\mathbf{x}), \quad \text { in } \Omega^{c}, \\
w=0, \quad \text { on } \Gamma_{0}, \\
w(\mathbf{x})=\mathcal{O}(1), \quad \text { as }|\mathbf{x}| \longrightarrow \infty
\end{gathered}
$$

Then, we let $x=\sqrt{\alpha} \xi$ and $y=\sqrt{\beta} \eta$. Problem (43) becomes

$$
\begin{gathered}
-\Delta w=-\left(\frac{\partial^{2} w}{\partial \xi^{2}}+\frac{\partial^{2} w}{\partial \eta^{2}}\right)=f(\mathbf{x}), \quad \text { in } \widetilde{\Omega}^{c}, \\
w=0, \quad \text { on } \widetilde{\Gamma}_{0}, \\
w(\mathbf{x})=\mathcal{O}(1), \quad \text { as }|\mathbf{x}| \longrightarrow \infty,
\end{gathered}
$$

where $\widetilde{\Omega}^{c}$ and $\widetilde{\Gamma}_{0}$ are the corresponding changes from $\Omega^{c}$ and $\Gamma_{0}$, respectively. Let $g$ be extended to $\widetilde{\Omega}^{c}, w=u-g, f=\Delta g$. Then, problem (44) is equivalent to

$$
\begin{gathered}
-\Delta u=0, \quad \text { in } \widetilde{\Omega}^{c}, \\
u=g, \quad \text { on } \widetilde{\Gamma}_{0}, \\
u(\mathbf{x})=\mathcal{O}(1), \quad \text { as }|\mathbf{x}| \longrightarrow \infty .
\end{gathered}
$$

Since it is difficult to estimate the convergence rate for a general unbounded domain $\Omega^{c}$, we here let $\Omega^{c}$ be an exterior domain of an ellipse $\Gamma_{0}$, with $\mu=\mu_{0}$, and $\Gamma_{1}$ is taken as stated in Section 1. We introduce the following conclusion first.

Lemma 2. If $u$ is the solution of

$$
\begin{array}{cc}
-\Delta u=0, & \text { in } \Omega_{1}, \\
u=u_{0}, & \text { on } \Gamma_{0}, \\
\frac{\partial u}{\partial n}=u_{n}, & \text { on } \Gamma_{1},
\end{array}
$$

where $\Omega_{1}$ is the elliptical ring domain between $\Gamma_{0}$ and $\Gamma_{1}$,

$$
\begin{aligned}
u_{0}= & \sum_{j=-\infty}^{+\infty} c_{j} \mathrm{e}^{\mathrm{i} j \phi} \in H^{1 / 2}\left(\Gamma_{0}\right), \\
u_{n}= & \frac{1}{\sqrt{J_{0}}}\left(\sum_{-\infty, j \neq 0}^{+\infty} d_{j}|j| \mathrm{e}^{\mathrm{i} j \phi}+d_{0}\right), \\
& \in H^{-1 / 2}\left(\Gamma_{1}\right),
\end{aligned}
$$

then there exist a unique $u \in H^{1}\left(\Omega_{1}\right)$ and

$$
\begin{aligned}
u(\mu, \phi)=\sum_{-\infty, j \neq 0}^{+\infty} & \left(\left(c_{j}\left(\mathrm{e}^{|j|\left(\mu-\mu_{1}\right)}+\mathrm{e}^{|j|\left(\mu_{1}-\mu\right)}\right)+d_{j}\right.\right. \\
& \left.\times\left(\mathrm{e}^{|j|\left(\mu-\mu_{0}\right)}-\mathrm{e}^{|j|\left(\mu_{0}-\mu\right)}\right)\right) \\
& \left.\times\left(\mathrm{e}^{|j|\left(\mu_{1}-\mu_{0}\right)}+\mathrm{e}^{|j|\left(\mu_{0}-\mu_{1}\right)}\right)^{-1}\right) \\
& \times \mathrm{e}^{\mathrm{i} j \phi}+c_{0}+d_{0}\left(\mu-\mu_{0}\right) .
\end{aligned}
$$

Proof. The result can be obtained directly from (46) by the separation of variables.

Theorem 3. If $0<\theta_{k}<1$, then the nonoverlapping domain decomposition method (7)-(9) is convergent.

Proof. We assume that the exact solution to problem (1) is $u$, and we let $\lambda=\left.u\right|_{\Gamma_{1}}, u_{k}=\left.u\right|_{\Omega_{k}}$, and $k=1,2$. Then, following (7)-(8), we let $e_{1}^{k}=\lambda-u_{1}^{k}$ and $\left.e_{1}^{k}\right|_{\Gamma_{1}}=\lambda-\lambda^{k} \dot{=} e_{2}^{k}$. From (24), we assume that $e_{2}^{k}=\sum_{j=-\infty}^{+\infty} a_{j} \mathrm{e}^{\mathrm{i} j \phi} \in H^{1 / 2}\left(\Gamma_{1}\right)$. By the natural integral equation (27), we have

$$
\frac{\partial e_{1}^{k}}{\partial n}=-\mathscr{K}_{1}\left(e_{2}^{k}\right)=-\frac{1}{\sqrt{J_{0}}} \sum_{j=-\infty}^{+\infty}|j| a_{j} \mathrm{e}^{\mathrm{i} j \phi} .
$$

So, $e_{1}^{k}$ satisfies

$$
\begin{gathered}
-\Delta e_{1}^{k}=0, \quad \text { in } \Omega_{1}, \\
e_{1}^{k}=0, \quad \text { on } \Gamma_{0}, \\
\frac{\partial e_{1}^{k}}{\partial n}=\frac{1}{\sqrt{J_{0}}} \sum_{j=-\infty}^{+\infty}|j| a_{j} \mathrm{e}^{\mathrm{i} j \phi}, \quad \text { on } \Gamma_{1} .
\end{gathered}
$$

By Lemma 2, one obtains

$$
e_{1}^{k}=-\sum_{j=-\infty}^{+\infty} a_{j} H_{j}(\mu) \mathrm{e}^{\mathrm{i} j \phi},
$$

with $H_{j}(\mu)=\left(\mathrm{e}^{|j|\left(\mu-\mu_{0}\right)}-\mathrm{e}^{|j|\left(\mu_{0}-\mu\right)}\right) /\left(\mathrm{e}^{|j|\left(\mu_{1}-\mu_{0}\right)}+\mathrm{e}^{|j|\left(\mu_{0}-\mu_{1}\right)}\right)$. From (51), $e_{1}^{k}$ confines on $\Gamma_{1}$ can be expressed as

$$
\begin{gathered}
\left.e_{1}^{k}\right|_{\Gamma_{1}}=-\sum_{j=-\infty}^{+\infty} a_{j} H_{j}\left(\mu_{1}\right) \mathrm{e}^{\mathrm{i} j \phi}, \\
\mathscr{K}_{1}\left(e_{1}^{k}\right)=-\frac{1}{\sqrt{J_{0}}} \sum_{j=-\infty}^{+\infty}|j| a_{j} H_{j}\left(\mu_{1}\right) \mathrm{e}^{\mathrm{i} j \phi} .
\end{gathered}
$$

Then, we have

$$
\begin{aligned}
\frac{\partial e_{1}^{k+1}}{\partial n} & =-\mathscr{K}_{1}\left(\lambda-\lambda^{k+1}\right) \\
& =\mathscr{K}_{1}\left(\theta_{k} u_{1}^{k}+\left(1-\theta_{k}\right) \lambda^{k}-\lambda\right) \\
& =-\theta_{k} \mathscr{K}_{1}\left(e_{1}^{k}\right)-\left(1-\theta_{k}\right) \mathscr{K}_{1}\left(e_{2}^{k}\right) \\
& =\frac{1}{\sqrt{J_{0}}} \sum_{j=-\infty}^{+\infty}|j| a_{j}\left(\theta_{k} H_{j}\left(\mu_{1}\right)-1+\theta_{k}\right) \mathrm{e}^{\mathrm{i} j \phi} .
\end{aligned}
$$


Let $E^{m} \doteq\left\|\partial e_{1}^{k} / \partial n\right\|_{-1 / 2, \Gamma_{1}}^{2}$, then $E^{m}=2 \pi \sum_{j=-\infty}^{+\infty}\left(j^{2} /\right.$ $\left.\sqrt{1+j^{2}}\right)\left|a_{j}\right|^{2}$ and

$$
\begin{aligned}
E^{m+1}= & 2 \pi \sum_{j=-\infty}^{+\infty} \frac{j^{2}}{\sqrt{1+j^{2}}}\left|a_{j}\right|^{2}\left(\theta_{k} H_{j}\left(\mu_{1}\right)-1+\theta_{k}\right)^{2} \\
= & \left(1-\theta_{k}\right)^{2} E^{m}+2 \pi \sum_{j=-\infty}^{+\infty} \frac{j^{2}}{\sqrt{1+j^{2}}}\left|a_{j}\right|^{2} \\
& \times \theta_{k} H_{j}\left(\mu_{1}\right)\left[\theta_{k}\left(H_{j}\left(\mu_{1}\right)+2\right)-2\right] .
\end{aligned}
$$

Assuming that $\delta_{1}=\inf _{j \in \mathbb{Z} /\{0\}} 2 /\left(2+H_{j}\left(\mu_{1}\right)\right)$, then $1>$ $\delta_{1} \geq 2 / 3$.

If $0<\theta_{k} \leq \delta_{1}, k=0,1,2, \ldots$, then

$$
E^{m+1}<\left(1-\theta_{k}\right)^{2} E^{m},
$$

or equally

$$
E^{m+1}<\prod_{j=1}^{m}\left(1-\theta_{j}\right)^{2} E^{1} \leq r^{m} E^{1}, \quad \frac{1}{9} \leq r<1 .
$$

By the trace theorem, we have

$$
\left\|e_{1}^{k}\right\|_{1, \Omega_{1}}^{2} \leq C E^{m} \longrightarrow 0, \quad m \longrightarrow \infty
$$

From (54), one also has

$$
\begin{aligned}
E^{m+1}= & 2 \pi \sum_{j=-\infty}^{+\infty} \frac{j^{2}}{\sqrt{1+j^{2}}}\left|a_{j}\right|^{2}\left(\theta_{k} H_{j}\left(\mu_{1}\right)-1+\theta_{k}\right)^{2} \\
= & \left(1-2 \theta_{k}\right)^{2} E^{m}+2 \pi \sum_{j=-\infty}^{+\infty} \frac{j^{2}}{\sqrt{1+j^{2}}}\left|a_{j}\right|^{2} \\
& \times \theta_{k} I_{j}\left(\mu_{1}\right)\left[\theta_{k}\left(I_{j}\left(\mu_{1}\right)-2\right)+1\right],
\end{aligned}
$$

with $I_{j}\left(\mu_{1}\right)=\left(1-H_{j}\left(\mu_{1}\right)\right) / 2$. Assuming that $\delta_{2}=$ $\sup _{j \in \mathbb{Z} /\{0\}} 1 /\left(2-I_{j}\left(\mu_{1}\right)\right)$, then $0<\delta_{2} \leq 2 / 3$.

For $\delta_{2} \leq \theta_{k}<1, k=0,1,2, \ldots$, the convergence result can be obtained similarly with (55)-(57). Therefore, for $0<$ $\theta_{k}<1$, the nonoverlapping domain decomposition method is convergent.

3.3. Discrete Nonoverlapping Alternating Algorithm and Convergence Analysis. Divide the $\operatorname{arc} \Gamma_{\mu_{1}}$ into $M$ parts, and take a finite element subdivision in $\Omega_{1}$ such that their nodes on $\Gamma_{\mu_{1}}$ are coincident. That is, we make a regular and quasiuniform triangulation $\mathscr{T}_{h}$ on $\Omega_{1}$, such that

$$
\Omega_{1}=\bigcup_{K \in \mathscr{T}_{h}} K
$$

where $K$ is a (curved) triangle and $h$ is the maximal diameter of triangles. Let

$$
V_{h}=\left\{v_{h} \in V|v|_{K} \text { is a linear polynomial, } \forall K \in \mathscr{T}_{h}\right\} .
$$

Then, the approximate problem of (39) can be written as follows

$$
\begin{aligned}
& \text { Find } u_{h} \in V_{h} \text {, such that } \\
& D\left(u_{h} ; u_{h}, v_{h}\right)+\widehat{D}\left(u_{h} ; u_{h}, v_{h}\right)=F\left(v_{h}\right), \quad \forall v_{h} \in V_{h},
\end{aligned}
$$

with

$$
\begin{gathered}
D\left(t_{h} ; u_{h}, v_{h}\right)=\int_{\Omega_{1}} a\left(\mathbf{x}, t_{h}\right)\left(\alpha \frac{\partial u_{h}}{\partial x} \frac{\partial v_{h}}{\partial x}+\beta \frac{\partial u_{h}}{\partial y} \frac{\partial v_{h}}{\partial y}\right) d \mathbf{x} \\
\widehat{D}\left(t_{h} ; u_{h}, v_{h}\right)=\sum_{j=1}^{+\infty} \frac{\sqrt{\alpha \beta}}{j \pi} \int_{0}^{2 \pi} \int_{0}^{2 \pi} a_{0}\left(t_{h}\left(\mu_{1}, \varphi^{\prime}\right)\right) \\
\times \frac{\partial u_{h}\left(\mu_{1}, \varphi^{\prime}\right)}{\partial \varphi^{\prime}} \frac{\partial v_{h}\left(\mu_{1}, \varphi\right)}{\partial \varphi} \\
\cdot \cos j\left(\varphi^{\prime}-\varphi\right) d \varphi^{\prime} d \varphi
\end{gathered}
$$

In practice, the sum of (63) is truncated to a finite number of terms $N$. By the hypothesis of $a(\cdot, \cdot)$, it is not difficult to know the following result.

Lemma 4. There exists a constant $C_{2}>0$ which has a different meaning in a different place and is related to $\alpha$ and $\beta$, such that

$$
\begin{aligned}
& |D(u ; u, v)+\widehat{D}(u ; u, v)| \leq C_{2}\|u\|_{1, \Omega_{1}}\|v\|_{1, \Omega_{1}}, \\
& D(u ; u, u)+\widehat{D}(u ; u, u) \geq C_{0}\|u\|_{1, \Omega_{1}}^{2}, \quad \forall u, v \in V .
\end{aligned}
$$

Proof. From (3) and (40), one can obtain that

$$
\begin{aligned}
& |D(u ; u, v)| \leq C_{2}^{\prime}\|u\|_{1, \Omega_{1}}\|v\|_{1, \Omega_{1}}, \\
& D(u ; u, u) \geq C_{0}\|u\|_{1, \Omega_{1}}^{2}, \quad \forall u, v \in V .
\end{aligned}
$$

For the natural integral equation, one can obtain the following result

$$
\begin{aligned}
& |\widehat{D}(u ; u, v)| \leq C_{2}^{\prime \prime}\|u\|_{1, \Omega_{1}}\|v\|_{1, \Omega_{1}}, \\
& \widehat{D}(u ; u, u) \geq 0, \quad \forall u, v \in V .
\end{aligned}
$$

For this purpose, for $u, v \in V$, we assume that

$$
\begin{aligned}
& u\left(\mu_{1}, \varphi^{\prime}\right)=\sum_{j=-\infty}^{+\infty} u_{j} \mathrm{e}^{\mathrm{i} j \varphi^{\prime}}, \\
& v\left(\mu_{1}, \varphi\right)=\sum_{j=-\infty}^{+\infty} v_{j} \mathrm{e}^{\mathrm{i} j \varphi},
\end{aligned}
$$

with $u_{j}=(1 / 2 \pi) \int_{0}^{2 \pi} u\left(\mu_{1}, \varphi^{\prime}\right) \mathrm{e}^{-\mathrm{i} j \varphi^{\prime}} d \varphi^{\prime}, v_{j}=(1 / 2 \pi) \int_{0}^{2 \pi} v\left(\mu_{1}\right.$, $\varphi) \mathrm{e}^{-\mathrm{i} j \varphi} d \varphi$. Then, we have

$$
\begin{aligned}
& \frac{\partial u}{\partial \varphi^{\prime}}\left(\mu_{1}, \varphi^{\prime}\right)=\sum_{j=-\infty}^{+\infty} \mathrm{i} j u_{j} \mathrm{e}^{\mathrm{i} j \varphi^{\prime}}, \\
& \frac{\partial v}{\partial \varphi}\left(\mu_{1}, \varphi\right)=\sum_{j=-\infty}^{+\infty} \mathrm{i} j v_{j} \mathrm{e}^{\mathrm{i} j \varphi} .
\end{aligned}
$$


Combining property (3), Cauchy's inequality, and the trace theorem, we have

$$
\begin{aligned}
|\widehat{D}(u ; u, v)| \leq & C_{2}^{\prime \prime}\left(\sum_{j=-\infty}^{+\infty}|j| \cdot\left|u_{j}\right|^{2}\right)^{1 / 2} \\
& \times\left(\sum_{j=-\infty}^{+\infty}|j| \cdot\left|v_{j}\right|^{2}\right)^{1 / 2} \\
\leq & C_{2}^{\prime \prime}\left(\sum_{j=-\infty}^{+\infty}\left(1+j^{2}\right)^{1 / 2} \cdot\left|u_{j}\right|^{2}\right)^{1 / 2} \\
& \times\left(\sum_{j=-\infty}^{+\infty}\left(1+j^{2}\right)^{1 / 2} \cdot\left|v_{j}\right|^{2}\right)^{1 / 2} \\
\leq & C_{2}^{\prime \prime}\|u\|_{1 / 2, \Gamma_{\mu_{1}}}\|v\|_{1 / 2, \Gamma_{\mu_{1}}} \\
\leq & C_{2}^{\prime \prime}\|u\|_{1, \Omega_{1}}\|v\|_{1, \Omega_{1}}, \quad \forall u, v \in V .
\end{aligned}
$$

Next, we show that $\widehat{D}(u ; u, u) \geq 0$, for any $u \in V$. For any given $v \in V$, let us consider the following auxiliary problem in $\Omega_{1}$ :

$$
\begin{gathered}
-\nabla \cdot(a(\mathbf{x}, u) \nabla u)=0, \quad \text { in } \Omega_{1}, \\
u=0, \quad \text { on } \Gamma_{0}, \\
u=v, \quad \text { on } \Gamma_{\mu_{1}} .
\end{gathered}
$$

From the analysis in Section 2.1, we know that the solution $u$ to problem (70) satisfies

$$
\left.a_{0}(u) \frac{\partial u}{\partial n}\right|_{\Gamma_{\mu_{1}}}=\mathscr{K}_{1}\left(u\left(\mu_{1}, \varphi\right)\right)=\mathscr{K}_{1}\left(v\left(\mu_{1}, \varphi\right)\right) .
$$

Multiplying (70) by $u$ and integrating over $\Omega_{1}$, we have

$$
\widehat{D}(u ; u, u)=\int_{\Omega_{1}} a_{0}(u)|\nabla u|^{2} d \mathbf{x} \geq 0 .
$$

Letting $C_{2}=C_{2}^{\prime}+C_{2}^{\prime \prime}$, we can get the desired result.

We are now in the position to show the existence and uniqueness result for this type of problem (1). We begin with the following estimate.

Lemma 5. Any solution u to problem (39) satisfies

$$
\|u\|_{1, \Omega_{1}} \leq C_{3}\|f\|_{L^{2}\left(\Omega_{1}\right)}
$$

with $C_{3}$ a positive constant.

Proof. Taking $v$ equal to $u$ in (39), by Lemma 4, one has

$$
C_{0}\|u\|_{1, \Omega_{1}}^{2} \leq D(u ; u, u)+\widehat{D}(u ; u, u)=L(u) .
$$

Then, the desired result follows from Cauchy-Schwarz and Poincaré-Friedrichs inequalities.
Theorem 6. Problem (39) is uniquely solvable.

Proof. Since the space $V$ is separable (indeed, it is a closed subspace of the space $H^{1}\left(\Omega_{1}\right)$ which is separable), there exist increasing sequences of finite-dimensional Hilbert subspaces $V^{m}$ of $V$ such that

$$
V=\bigcup_{m \geq 0} V^{m}
$$

We define a mapping $\Phi_{m}: V^{m} \rightarrow V^{m}$ by

$$
\begin{aligned}
\left(\Phi_{m}(u), v\right)= & D(u ; u, v)+\widehat{D}(u ; u, v) \\
& -L(v), \quad \forall u, v \in V^{m},
\end{aligned}
$$

where $(\cdot, \cdot)$ stands for the scalar product on $V^{m} \times V^{m}$. Since the function $a(\mathbf{x}, u)$ is bounded and the trace of the function in $V$ on $\Gamma_{1}$ belongs to $H^{1 / 2}\left(\Gamma_{1}\right)$, hence at least to $L^{4}\left(\Gamma_{1}\right)$ from the Sobolev embedding, each mapping $\Phi_{m}$ is well defined and continuous on $V^{m}$. What's more, by Lemmas 4 and 5, we obtain

$$
\begin{aligned}
\left(\Phi_{m}(u), u\right) & =D(u ; u, u)+\widehat{D}(u ; u, u)-L(u) \\
& \geq C_{0}\|u\|_{1, \Omega_{1}}^{2}-C_{3}\|f\|_{L^{2}\left(\Omega_{1}\right)}\|u\|_{1, \Omega_{1}}
\end{aligned}
$$

So, the right-hand side is nonnegative on the circle of radius $\mu$ which is defined by

$$
\mu=\frac{C_{3}}{C_{0}}\|f\|_{L^{2}\left(\Omega_{1}\right)} .
$$

Applying Brouwer's fixed point theorem [21] yields the existence of $u^{m}$ in $V^{m}$, with norm less than $\mu$, such that

$$
\Phi_{m}\left(u^{m}\right)=0
$$

Since the sequence $\left\{u^{m}\right\}_{m}$ is bounded by $\mu$ in $V$, there exists a subsequence which converges weakly to $u$ in $V^{m}$. Using the compactness of the imbedding of $H^{1 / 2}\left(\Gamma_{1}\right)$ into $L^{3}\left(\Gamma_{1}\right)$, we obtain that $u$ is a solution to problem (39).

Now, we show the uniqueness of the solution. Let $u$ and $\bar{u}$ be two solutions to this problem. Then, taking $v$ equal to $u$ and $\bar{u}$ in (39), respectively, and combining with Lemma 4, one obtains

$$
\begin{aligned}
C_{2}\|u\|_{1, \Omega_{1}}\|\bar{u}\|_{1, \Omega_{1}} & \geq D(\bar{u} ; \bar{u}, u)+\widehat{D}(\bar{u} ; \bar{u}, u) \\
& =D(u ; u, u)+\widehat{D}(u ; u, u) \\
& \geq C_{0}\|u\|_{1, \Omega_{1}}^{2}, \\
C_{2}\|u\|_{1, \Omega_{1}}\|\bar{u}\|_{1, \Omega_{1}} & \geq D(u ; u, \bar{u})+\widehat{D}(u ; u, \bar{u}) \\
& =D(\bar{u} ; \bar{u}, \bar{u})+\widehat{D}(\bar{u} ; \bar{u}, \bar{u}) \geqslant C_{0}\|\bar{u}\|_{1, \Omega_{1}}^{2} .
\end{aligned}
$$

Since $C_{2}>C_{0}$, combining (80) with (81), we deduce the desired result. 


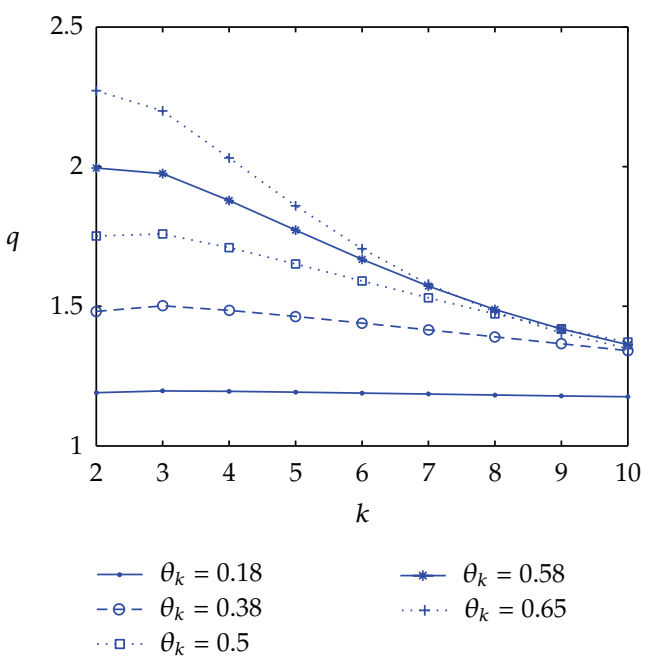

(a)

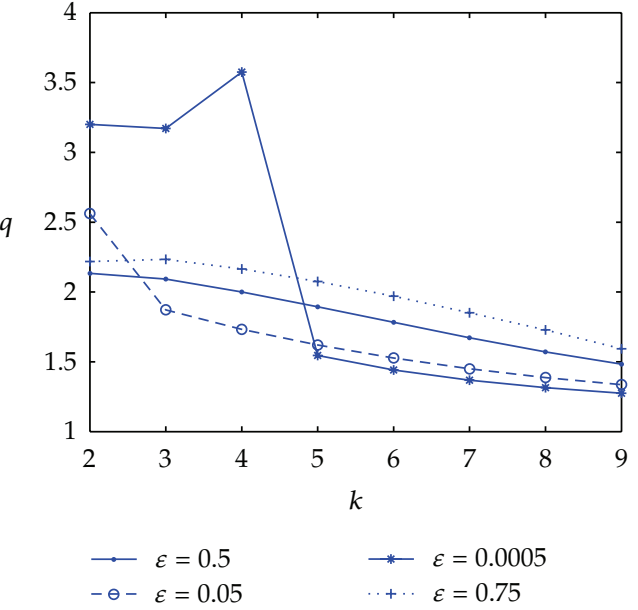

(b)

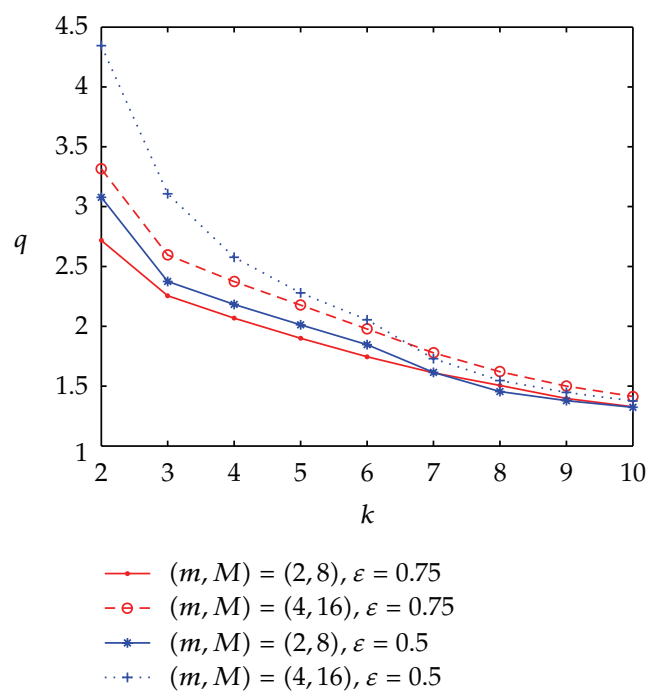

(c)

FIGURE 2: (a) The relationship between $\theta_{k}$ and convergence rate $(N=10, m=4$ and $M=16)$. (b) The relationship between $\varepsilon$ and convergence rate $\left(N=10, m=4, M=16\right.$ and $\left.\theta_{k}=0.58\right)$. (c) The relationship between meshes and convergence rate $\left(N=10, \theta_{k}=0.65\right)$.

From the discrete problem (61), similar to the discussion for the linear problem in $[6,8]$, we can get a system of algebraic equations for our quasilinear problem with the following form:

$$
\left(\begin{array}{cc}
A_{11}+K_{h} & A_{12} \\
A_{21} & A_{22}
\end{array}\right)\left(\begin{array}{l}
\mathbf{U} \\
\mathbf{V}
\end{array}\right)=\left(\begin{array}{l}
\mathbf{0} \\
\mathbf{b}
\end{array}\right)
$$

where $\mathbf{U}$ is a vector whose components are function values at nodes on $\Gamma_{1}$, and $\mathbf{V}$ is a vector whose components are function values at interior nodes of $\Omega_{1}$. The matrix $A \doteq A(u)=\left(\begin{array}{ll}A_{11} & A_{12} \\ A_{21} & A_{22}\end{array}\right)$ is the stiffness matrix obtained from finite element in $\Omega_{1}$, while $K_{h} \doteq K_{h}\left(\left.u\right|_{\Gamma_{1}}\right)$ follows from the natural boundary element method on $\Gamma_{1}$.

Problem (82) can also be rewritten as follows:

$$
\left(\begin{array}{ll}
A_{11} & A_{12} \\
A_{21} & A_{22}
\end{array}\right)\left(\begin{array}{l}
\mathbf{U} \\
\mathbf{V}
\end{array}\right)=\left(\begin{array}{c}
-K_{h} \mathbf{U} \\
\mathbf{b}
\end{array}\right)
$$

Then, we have the iterative algorithm

$$
\left(\begin{array}{ll}
A_{11} & A_{12} \\
A_{21} & A_{22}
\end{array}\right)\left(\begin{array}{l}
\mathbf{U}_{k} \\
\mathbf{V}_{k}
\end{array}\right)=\left(\begin{array}{c}
-K_{h} \Lambda_{k} \\
\mathbf{b}
\end{array}\right)
$$

with

$$
\Lambda_{k+1}=\theta_{k} \mathbf{U}_{k}+\left(1-\theta_{k}\right) \Lambda_{k}, \quad k=0,1, \ldots
$$

By condition (3), one obtains that $A$ is a positive definite matrix, so we know that $A_{22}^{-1}$ exists. Now, we let $S_{h}=S_{h}^{(1)}+K_{h}$ be the discrete analogue of the Steklov-Poincaré operator on $\Gamma_{1}$, with $S_{h}^{(1)}=A_{11}-A_{12} A_{22}^{-1} A_{21}$ and $\mathbf{B}=-A_{12} A_{22}^{-1} \mathbf{b}$. Then, similar to the proof of Theorems 7.6 and 7.7 in [6], we have that the alternating algorithm (84)-(85) is equivalent to the preconditioned Richardson iteration:

$$
S_{h}^{(1)}\left(\Lambda^{k+1}-\Lambda^{k}\right)=\theta_{k}\left(\mathbf{B}-S_{h} \Lambda^{k}\right) .
$$




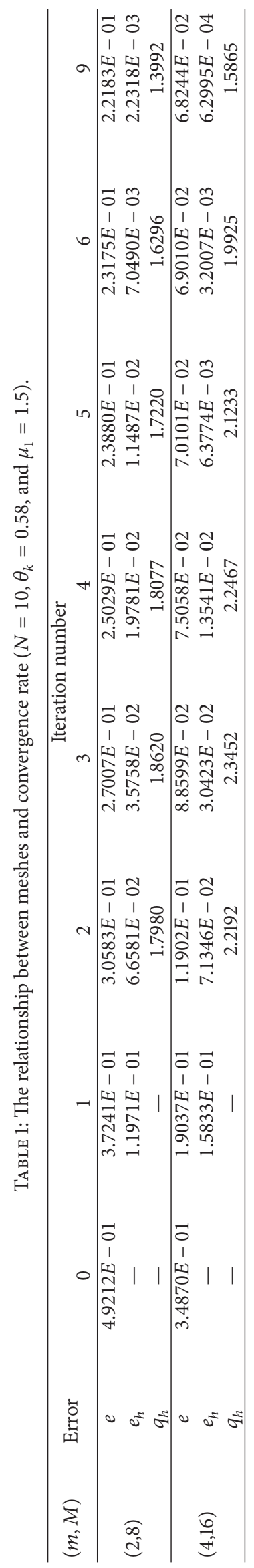




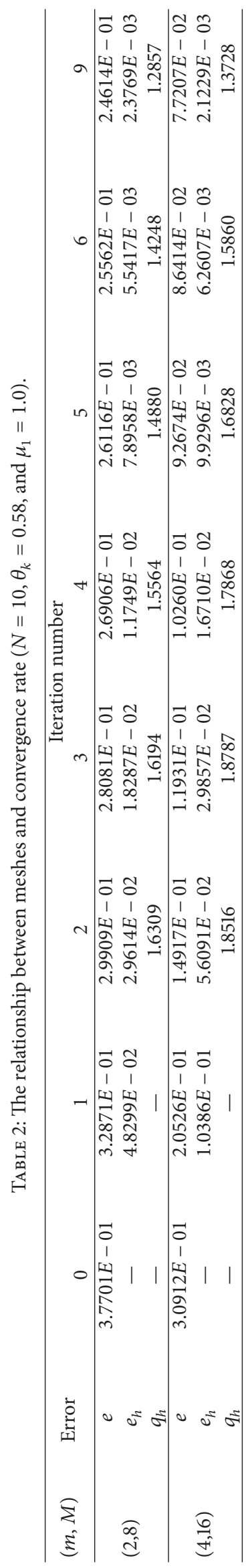




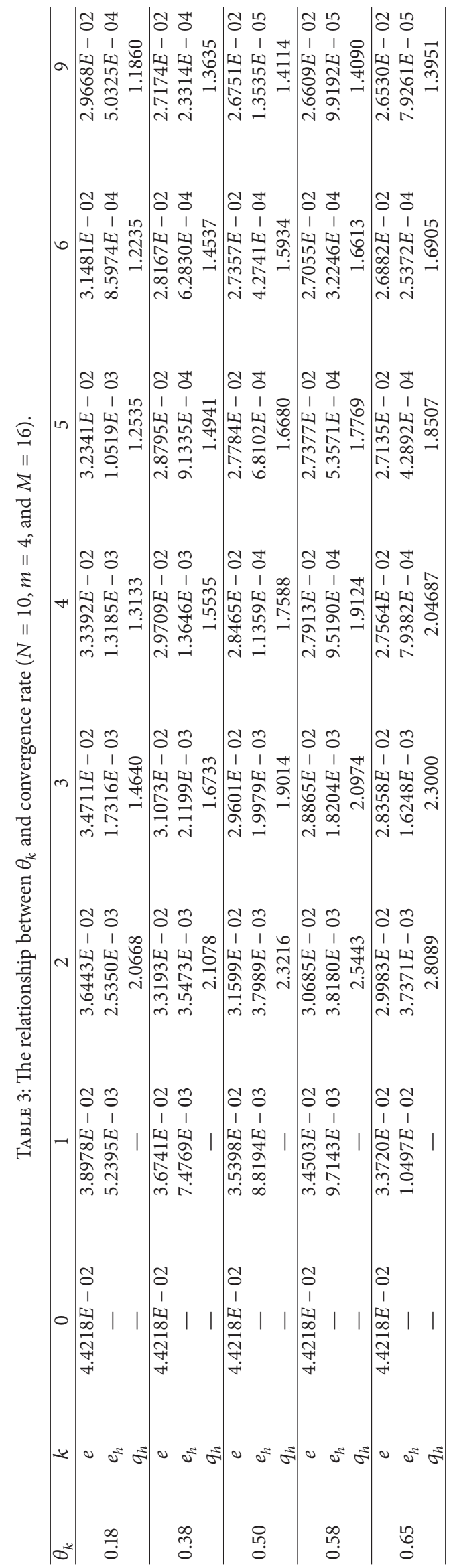




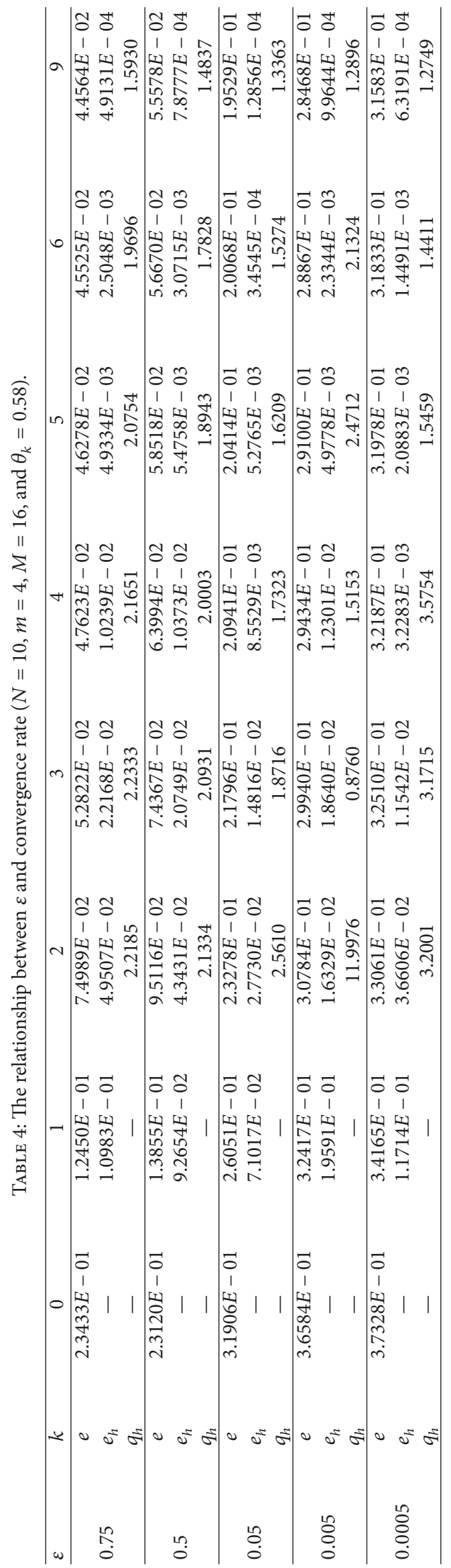




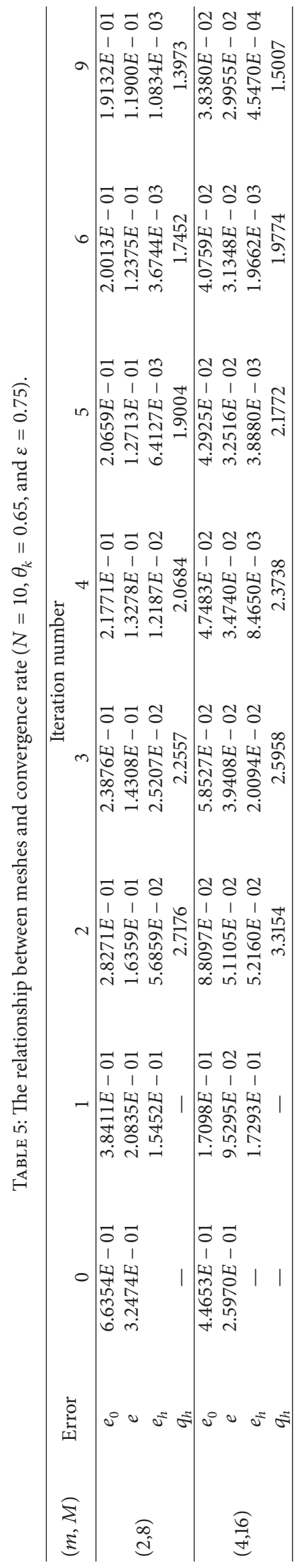




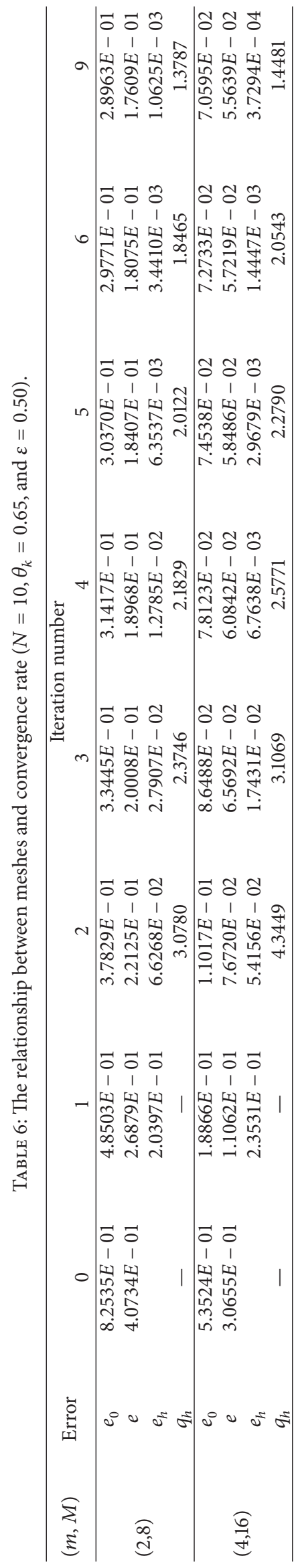


And we also have the following convergence result.

Theorem 7. If $0<\min \theta_{k} \leq \max \theta_{k}<1$, then the discrete nonoverlapping alternating method (84)-(85) is convergent, and both the convergence rate and the condition number of $\left[S_{h}^{(1)}\right]^{-1} S_{h}$ are independent of the mesh size $h$.

\section{Numerical Examples}

In this section, we will give some examples to confirm our theoretical results. In the following, we choose the finite element space as given in (60). For simplicity, we let

$$
\begin{aligned}
& \Delta \mu=\frac{\mu_{1}-\mu_{0}}{m}, \quad \Delta \varphi=\frac{2 \pi}{M}, \\
& e_{0}(k)=\left\|u-u_{h, N}^{k}\right\|_{L^{2}\left(\Omega_{i}\right)}, \\
& e(k)=\left\|u-u_{h, N}^{k}\right\|_{L^{\infty}\left(\Omega_{i}\right)} .
\end{aligned}
$$

And we let $e_{h}(k)$ denote the maximal error between the iteration $k-1$ and $k$, that is, $e_{h}(k)=\left\|u_{h, N}^{k}-u_{h, N}^{k-1}\right\|_{L^{\infty}\left(\Omega_{i}\right)}$ and $q_{h}(k)=e_{h}(k-1) / e_{h}(k)$ simulate the convergence rate.

We give the following four examples. Examples 8 and 11 show the relationship between meshes and convergence rate for the cases $\alpha=\beta=1$ and $\beta>\alpha>0$, respectively. Example 9 focuses on the effect of the relaxation factor $\theta_{k}$ to the convergence rate. And Example 10 wants to show the relationship between the coefficients $\alpha, \beta$ and the convergence rate.

Example 8. We assume the exterior domain $\Omega^{c}$ with elliptical boundary $\Gamma_{0}=\left\{\left(\mu_{0}, \varphi\right) \mid \mu_{0}=0.8,0 \leq \varphi \leq 2 \pi\right\}, \Gamma_{\mu_{1}}=$ $\left\{\left(\mu_{1}, \varphi\right) \mid \mu_{1}>\mu_{0}, 0 \leq \varphi \leq 2 \pi\right\}$. Now, we consider the problem

$$
\begin{gathered}
-\nabla \cdot(a(\mathbf{x}, u) \nabla u)=f, \quad \text { in } \Omega_{1}, \\
u=0, \quad \text { on } \Gamma_{0}, \\
a_{0}(u) \frac{\partial u}{\partial n}=\mathscr{K}_{1}\left(u\left(\mu_{1}, \varphi\right)\right), \quad \text { on } \Gamma_{\mu_{1}},
\end{gathered}
$$

when $a(\mathbf{x}, u)=1 /\left(1+u^{2}\right), f=0$, and $f_{0}=1.35$.

The exact solution of Example 8 is $u=\tan (2 \sinh \mu$ $\left.\sin \varphi / f_{0}(\cosh 2 \mu+\cos 2 \varphi)\right)$. For different meshes and $\mu_{1}$, we have the following results ranked in Tables 1 and 2 .

Example 9. Similar with Example 8, $\Gamma_{0}$ and $a(\mathbf{x}, u)$ are replaced by $\Gamma_{0}=\left\{\left(\mu_{0}, \varphi\right) \mid \mu_{0}=0.5,0 \leq \varphi \leq 2 \pi\right\}, \Gamma_{1}=$ $\left\{\left(\mu_{1}, \varphi\right) \mu_{1}=1.0,0 \leq \varphi \leq 2 \pi\right\}$, and $a(\mathbf{x}, u)=1 / \sqrt{1-u^{2}}$, respectively.

The exact solution of Example 9 is $u=\sin (2 \cosh \mu$ $\left.\cos \varphi / f_{0}(\cosh 2 \mu+\cos 2 \varphi)\right)$. The numerical results are given in Table 3 and Figure 2(a).
Example 10. We assume the exterior domain $\Omega^{c}$ with elliptical boundary $\Gamma_{0}=\left\{\left(\mu_{0}, \varphi\right) \mid \mu_{0}=0.8,0 \leq \varphi \leq 2 \pi\right\}, \Gamma_{\mu_{1}}=$ $\left\{\left(\mu_{1}, \varphi\right) \mid \mu_{1}=2.0,0 \leq \varphi \leq 2 \pi\right\}$. Now, we consider the problem

$$
-\left(\frac{\partial}{\partial x}\left(\varepsilon a(\mathbf{x}, u) \frac{\partial u}{\partial x}\right)+\frac{\partial}{\partial y}\left(a(\mathbf{x}, u) \frac{\partial u}{\partial y}\right)\right)=f(\mathbf{x}),
$$

in $\Omega_{i}$,

$$
\begin{gathered}
u=0, \quad \text { on } \Gamma_{0}, \\
\varepsilon n_{x} a_{0}(u) \frac{\partial u}{\partial x}+n_{y} a_{0}(u) \frac{\partial u}{\partial y} \\
=\mathscr{K}_{1}\left(u\left(\mu_{1}, \varphi\right)\right), \quad \text { on } \Gamma_{\mu_{1}}, \\
\text { when } a(\mathbf{x}, u)=1 /\left(1+u^{2}\right), f_{0}=1.25 \text { and } \\
f=\frac{2(1-\varepsilon) \sinh \mu \sin \varphi\left(3 \cosh ^{2} \mu \cos ^{2} \varphi-\sinh ^{2} \mu \sin ^{2} \varphi\right)}{(\cosh 2 \mu+\cos 2 \varphi)^{3}}
\end{gathered}
$$

The exact solution of Example 10 is $u=\tan (2 \sinh \mu$ $\left.\sin \varphi / f_{0}(\cosh 2 \mu+\cos 2 \varphi)\right)$. The numerical results are given in Table 4 and Figure 2(b).

Example 11. Similar with Example 10, $\Gamma_{0}, \Gamma_{1}$, and $a(\mathbf{x}, u)$ are replaced by $\Gamma_{0}=\left\{\left(\mu_{0}, \varphi\right) \mid \mu_{0}=0.5,0 \leq \varphi \leq 2 \pi\right\}, \Gamma_{1}=$ $\left\{\left(\mu_{1}, \varphi\right) \mu_{1}=1.5,0 \leq \varphi \leq 2 \pi\right\}$, and $a(\mathbf{x}, u)=1 / \sqrt{1-u^{2}}$, respectively. And we take

$$
f=\frac{2(1-\varepsilon) \cosh \mu \cos \varphi\left(\cosh ^{2} \mu \cos ^{2} \varphi-3 \sinh ^{2} \mu \sin ^{2} \varphi\right)}{(\cosh 2 \mu+\cos 2 \varphi)^{3}} .
$$

The exact solution of Example 11 is $u=\sin (2 \cosh \mu$ $\left.\cos \varphi / f_{0}(\cosh 2 \mu+\cos 2 \varphi)\right)$. The numerical results are given in Tables 5 and 6 and Figure 2(c).

From the numerical results, one obtains that the numerical errors can be affected by the choice of relaxation factor $\theta_{k}$, the coefficient $\varepsilon$, and the location of the artificial boundary. In Tables 1, 2, 5, and 6 and Figure 2(c), the relationship between the meshes and the convergence rate is presented. We obtain that the convergence rate is independent of the finite element mesh size. In Table 3 and Figure 2(c), the convergence rates for different relaxation factor $\theta_{k}$ are compared. The results indicate that the choice of the relaxation factor is very important for the performance of the nonoverlapping alternating method. On the other hand, the convergence rate is not sensitive to the relaxation factor $\theta_{k}$ between the interval $(0.5,0.67)$. What's more, as it is shown in Table 4 and Figure 2(c), for the anisotropic problems, the choice of $\varepsilon$ can also affect the convergence rate.

\section{Acknowlegments}

This work is supported by the National Natural Science Foundation of China, Contact/Grant no. 11071109 and the Foundation for Innovative Program of Jiangsu Province, Contact/Grant no. CXZZ12_0383 and CXZZ11_0870. 


\section{References}

[1] G. Ben-Porat and D. Givoli, "Solution of unbounded domain problems using elliptic artificial boundaries," Communications in Numerical Methods in Engineering, vol. 11, no. 9, pp. 735-741, 1995.

[2] Y. Boubendir, X. Antoine, and C. Geuzaine, "A quasi-optimal non-overlapping domain decomposition algorithm for the Helmholtz equation," Journal of Computational Physics, vol. 231, no. 2, pp. 262-280, 2012.

[3] Q. Y. Hu, S. Shu, and J. Wang, "Nonoverlapping domain decomposition methods with a simple coarse space for elliptic problems," Mathematics of Computation, vol. 79, no. 272, pp. 2059-2078, 2010.

[4] A. Siahaan, C. H. Lai, and K. Pericleous, "A nonoverlapping domain decomposition method for nonlinear physical processes," Proceedings in Applied Mathematics and Mechanics, vol. 7, pp. 2140003-2140004, 2007.

[5] Q. Du and D. Yu, "A domain decomposition method based on natural boundary reduction for nonlinear time-dependent exterior wave problems," Computing, vol. 68, no. 2, pp. 111-129, 2002.

[6] D.-H. Yu, Natural Boundary Integral Method and its Applications, vol. 539 of Mathematics and its Applications, Science Press \& Kluwer Academic Publishers, Dordrecht, The Netherlands, 2002.

[7] M. Yang and Q. Du, "A Schwarz alternating algorithm for elliptic boundary value problems in an infinite domain with a concave angle," Applied Mathematics and Computation, vol. 159, no. 1, pp. 199-220, 2004.

[8] D. H. Yu, "Domain decomposition method for unbounded domains," in Proceedings of the 8th International Conference on Domain Decomposition Methods, pp. 125-132, Beijing, China, 1997.

[9] Q. K. Du and D. H. Yu, "Domain decomposition methods based on natural boundary reduction for wave equation," Chinese Journal of Computational Physics, vol. 18, pp. 417-422, 2001.

[10] Q. Du and M. Zhang, "A non-overlapping domain decomposition algorithm based on the natural boundary reduction for wave equations in an unbounded domain," Numerical Mathematics, vol. 13, no. 2, pp. 121-132, 2004.

[11] H. Han, Z. Huang, and D. Yin, "Exact artificial boundary conditions for quasilinear elliptic equations in unbounded domains," Communications in Mathematical Sciences, vol. 6, no. 1, pp. 71-83, 2008.

[12] I. Hlaváček, M. Křížek, and J. Malý, "On Galerkin approximations of a quasilinear nonpotential elliptic problem of a nonmonotone type," Journal of Mathematical Analysis and Applications, vol. 184, no. 1, pp. 168-189, 1994.

[13] I. Hlaváček, "A note on the Neumann problem for a quasilinear elliptic problem of a nonmonotone type," Journal of Mathematical Analysis and Applications, vol. 211, no. 1, pp. 365-369, 1997.

[14] D. Liu and D. Yu, "A FEM-BEM formulation for an exterior quasilinear elliptic problem in the plane," Journal of Computational Mathematics, vol. 26, no. 3, pp. 378-389, 2008.

[15] S. Meddahi, M. González, and P. Pérez, "On a FEM-BEM formulation for an exterior quasilinear problem in the plane," SIAM Journal on Numerical Analysis, vol. 37, no. 6, pp. 18201837, 2000.

[16] D. N. Arnold, P. G. Ciarlet, and P. L. Lions, Nonlinear Partial Differential Equations and Their Applications, North-Holland, New York, NY, USA, 2002.
[17] J. B. Keller and M. J. Grote, "Exact non-reflecting boundary conditions," Journal of Computational Physics, vol. 82, pp. 172192, 1989.

[18] J. M. Wu and D. H. Yu, "The natural boundary element method for an exterior elliptic domain," Mathematica Numerica Sinica, vol. 22, no. 3, pp. 355-368, 2000 (Chinese).

[19] G. N. Gatica, G. C. Hsiao, and M. E. Mellado, "A domain decomposition method based on BEM and FEM for linear exterior boundary value problems," Journal of Mathematical Analysis and Applications, vol. 262, no. 1, pp. 70-86, 2001.

[20] D. B. Ingham and M. A. Kelmanson, Boundary Integral Equation Analyses of Singular, Potential, and Biharmonic Problems, vol. 7 of Lecture Notes in Engineering, Springer, Berlin, Germany, 1984.

[21] V. Girault and P.-A. Raviart, Finite Element Methods for NavierStokes Equations, Theory and Algorithms, vol. 5 of Springer Series in Computational Mathematics, Springer, Berlin, Germany, 1986. 


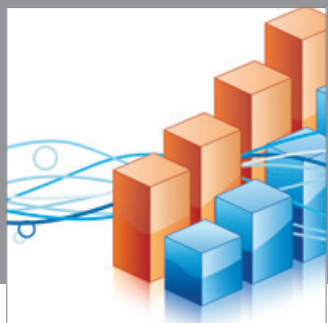

Advances in

Operations Research

mansans

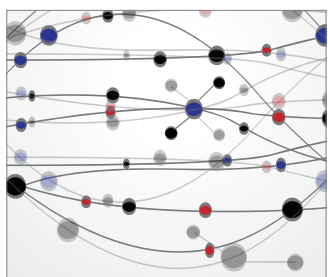

The Scientific World Journal
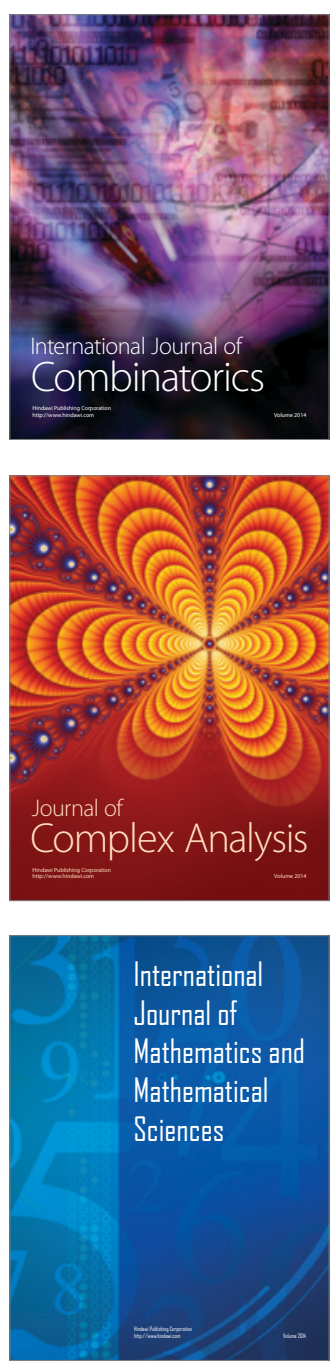
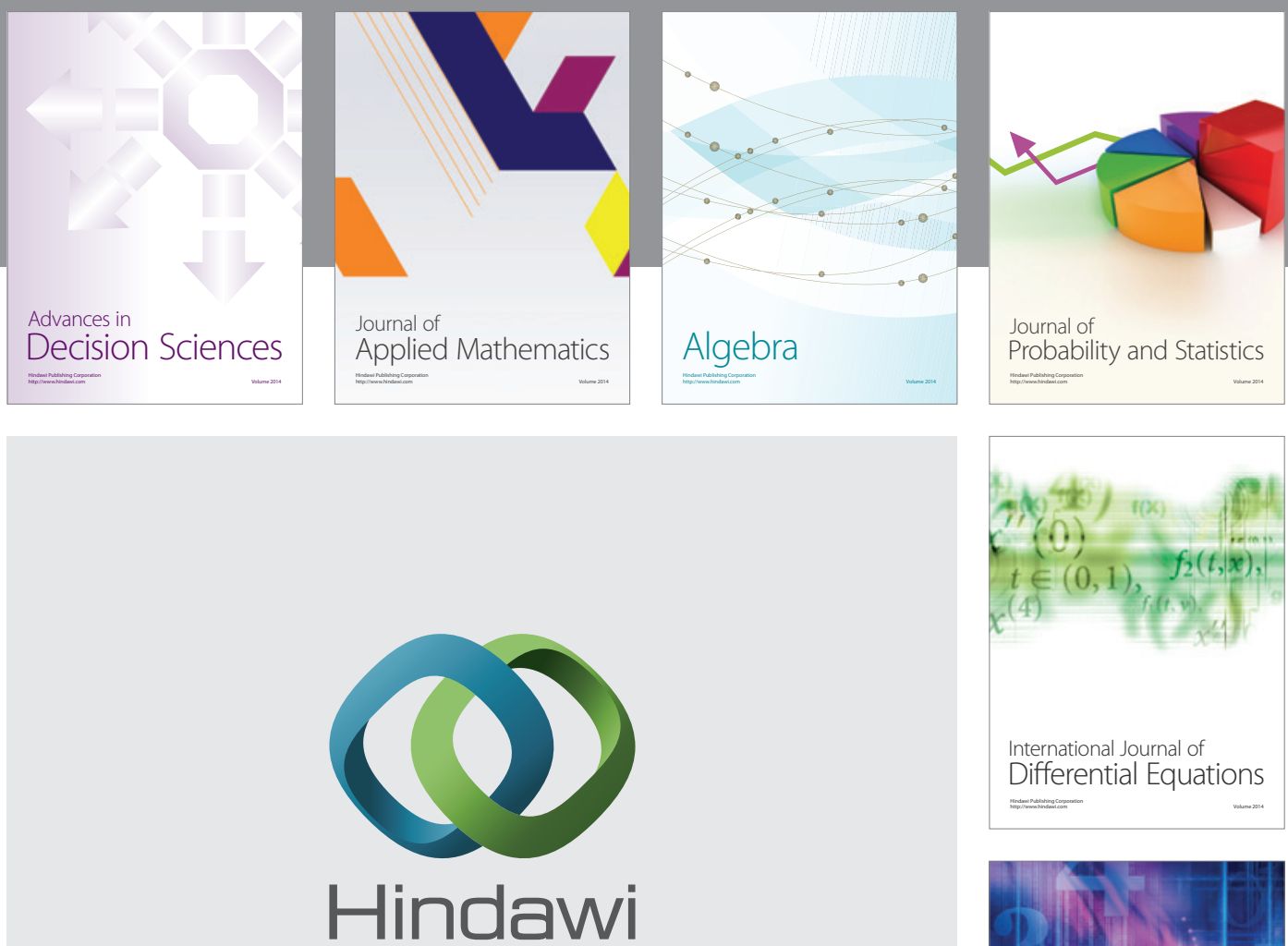

Submit your manuscripts at http://www.hindawi.com
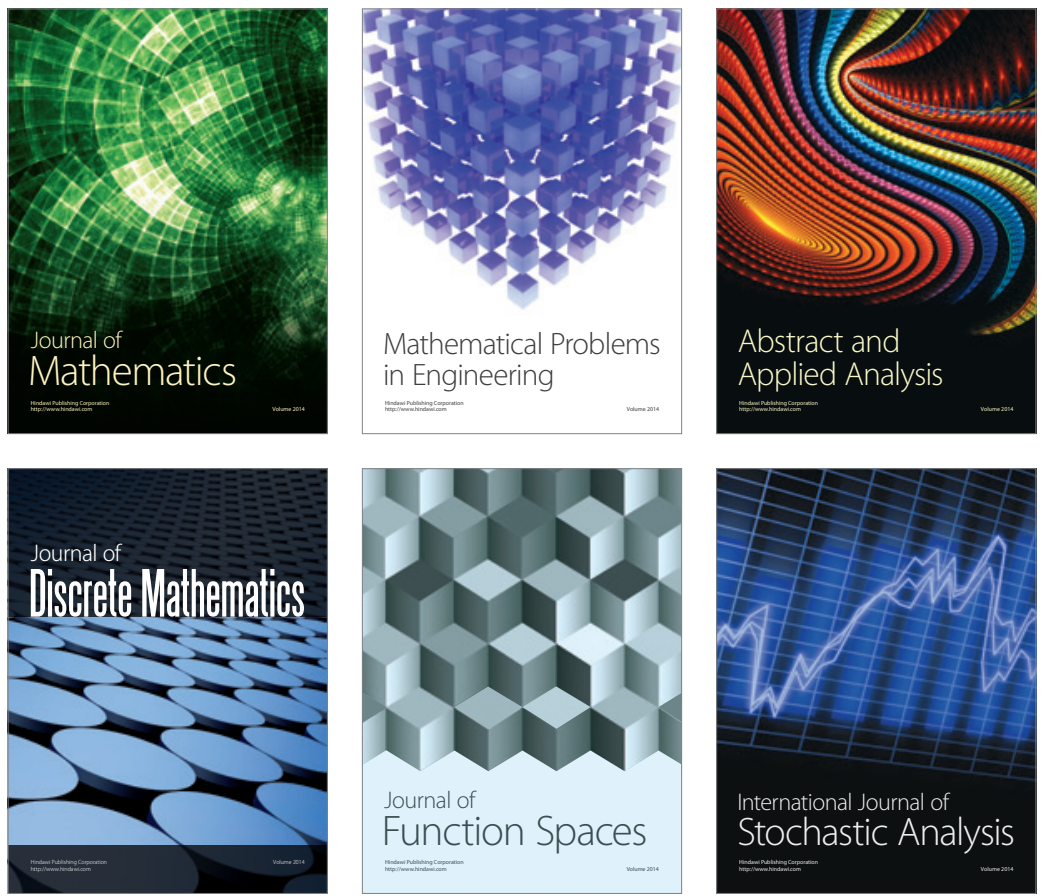

Journal of

Function Spaces

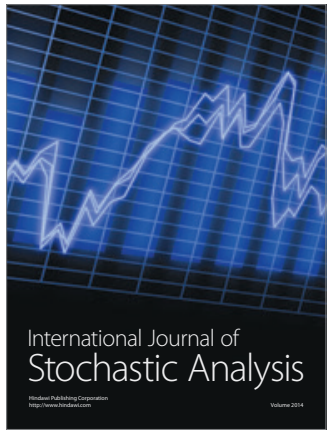

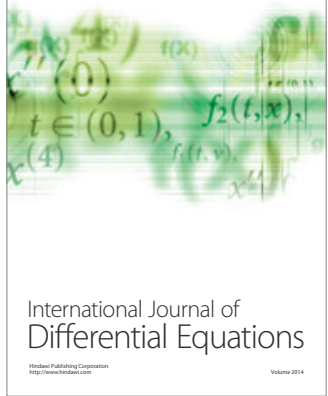
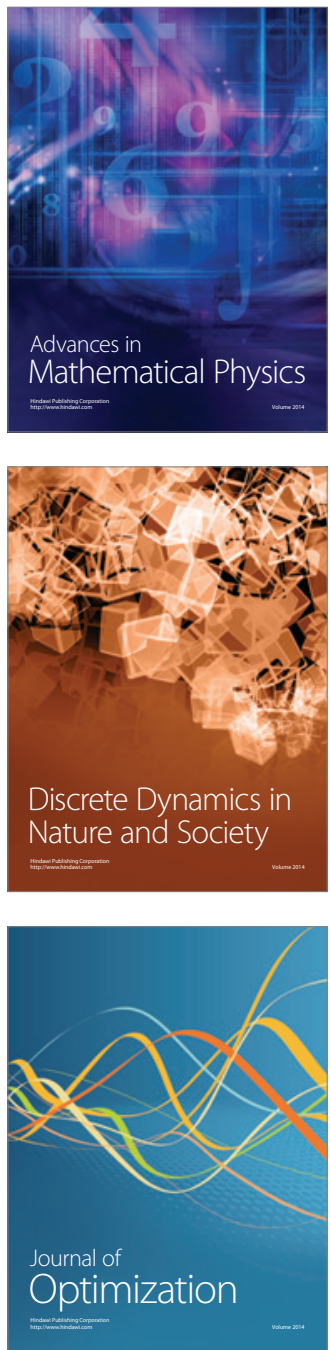